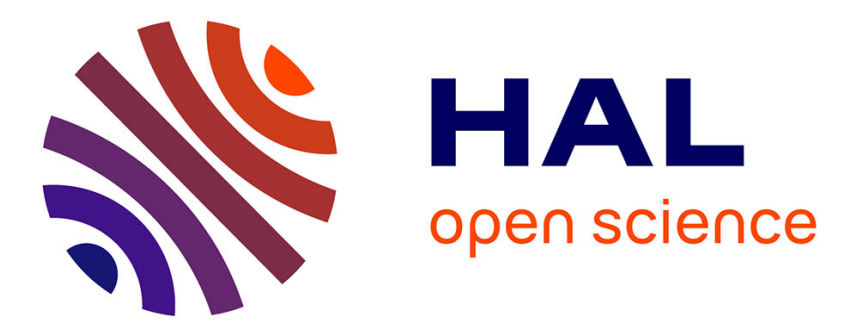

\title{
Experimental Nonlinear Identification of an Aircraft with Bolted Connections
}

\author{
G. de Filippis, J.P. Nöel, G. Kerschen, L. Soria, C. Stephan
}

\section{To cite this version:}

G. de Filippis, J.P. Nöel, G. Kerschen, L. Soria, C. Stephan. Experimental Nonlinear Identification of an Aircraft with Bolted Connections. IMAC XXXIV 2016, Jan 2016, ORLANDO, United States. p. 263-278, 10.1007/978-3-319-15221-9_24. hal-01436773

\section{HAL Id: hal-01436773 https://hal.science/hal-01436773}

Submitted on 23 Jan 2017

HAL is a multi-disciplinary open access archive for the deposit and dissemination of scientific research documents, whether they are published or not. The documents may come from teaching and research institutions in France or abroad, or from public or private research centers.
L'archive ouverte pluridisciplinaire HAL, est destinée au dépôt et à la diffusion de documents scientifiques de niveau recherche, publiés ou non, émanant des établissements d'enseignement et de recherche français ou étrangers, des laboratoires publics ou privés. 


\title{
Experimental Nonlinear Identification of an Aircraft with Bolted Connections
}

\author{
G. De Filippis ${ }^{1}$, J.P. Noël ${ }^{2}$, G. Kerschen ${ }^{2}$, L. Soria ${ }^{1}$, C. Stephan ${ }^{3}$ \\ ${ }^{1}$ Dipartimento di Meccanica, Matematica e Management \\ Politecnico di Bari, Italy \\ giovanni.defilippis, leonardo.soria@poliba.it \\ ${ }^{2}$ Space Structures and Systems Laboratory (S3L) \\ Aerospace and Mechanical Engineering Department \\ University of Liège, Liège, Belgium \\ jp.noel, g.kerschen@ulg.ac.be \\ ${ }^{3}$ ONERA - The French Aerospace Lab, France \\ cyrille.stephan@onera.fr
}

\begin{abstract}
Aircraft structures are known to be prone to nonlinear phenomena, especially as they constantly become lighter and hence more flexible. One specific challenge that is regularly encountered is the modeling of the mounting interfaces between aircraft subcomponents. Indeed, for large amplitudes of vibration, such interfaces may loosen and, in turn, trigger complex mechanisms such as friction and clearances. In this context, the present work intends to investigate the nonlinear dynamics of the Morane-Saulnier Paris aircraft, accessible at ONERA. This aircraft possesses multiple bolted connections between two external fuel tanks and wing tips. The objective of the paper is specifically to carry out an adequate identification of the numerous nonlinearities affecting the dynamics of this full-scale structure. Nonlinearity detection and the subsequent subspace-based parameter estimation have been performed on experimental data, collected during an on-ground test campaign. Nonlinearity detection is first achieved by the comparison of frequency response functions estimated at low excitation level, with those obtained at high amplitude level, yielding insight towards accurately characterizing the behavior of the bolted connections. Then, a nonlinear subspace identification method is applied to measured data to estimate the linear and nonlinear parameters of the structure and novel strategies and tools that overcome specific arisen problems are developed.
\end{abstract}

Keywords: Nonlinear system identification; frequency-domain subspace method; full-scale aircraft; cubic splines; spurious poles.

\section{INTRODUCTION}

The increasing levels of performance demanded to aircraft structures can require the implementation of design procedures and options that may, in turn, lead to the activation of some nonlinear phenomena. Numerous works reporting on the evidence of existing nonlinearities in aerospace applications can be found in the technical literature, as, to give only some examples, Ref. ${ }^{[1-3]}$. Classical linear subspace-based methods ${ }^{[4]}$ have recently been generalized to handle these occurrences ${ }^{[5,6]}$. 
Specifically, the frequency-domain nonlinear subspace identification (FNSI) method has been proved to successfully deal with grey-box models, affected by many nonlinearities ${ }^{[7]}$.

In this paper, the FNSI methodology is applied for the first time to a full-scale structure, the Morane-Saulnier Paris aircraft. This aircraft presents multiple bolted connections between the wings and two external fuel tanks, located at the wing tips. The experimental identification requires that a considerable number of output locations are utilized on the aircraft main body and at the two the ends of the mounting interfaces, where nonlinear behaviors are expected to arise. Due to the presence of many nonlinearities, a relevant number of basis functions are included in the FNSI routine. The development of novel tools and strategies is carried out to overcome the problems related to the high dimensionality of the consequent inverse problem. Moreover, classical subspace methods do not provide the user with the possibility of selecting among the poles computed at a given model order. This means that, in the usual procedure, all the calculated poles have to be included, the physical as the spurious ones. To avoid this drawback, an appropriate strategy is employed, that allows for removing the undesired spurious poles.

Linear identification is first performed at low amplitude level, with the aim of collecting the modal parameters. Then, the comparison between frequency response functions (FRFs) estimated at low and at high amplitude level is employed for detecting the overall nonlinear behavior of the structure. Afterward, the FNSI method is applied to estimate the underlying linear properties and the nonlinear restoring forces. Finally, a novel strategy for spurious poles' removal is applied to the identified model.

\section{DESCRIPTION OF THE AIRCRAFT AND OF THE EXPERIMENTAL SETUP}

The structure is the airframe of the Morane-Saulnier Paris aircraft, represented in Figure 1. This French jet aircraft was built during the 1950s and was used as a trainer and liaison aircraft. The structural configuration under consideration corresponds to the aircraft without its jet engines and standing on the ground through its three landing gears with deflated tires. A specimen of this airplane is present in ONERA's laboratory. It is used for training engineers and technicians for ground vibration tests (GVT) and for research purposes. GVT exhibit nonlinear behavior in the connection between the wings and external fuel tanks located at the wing tip.

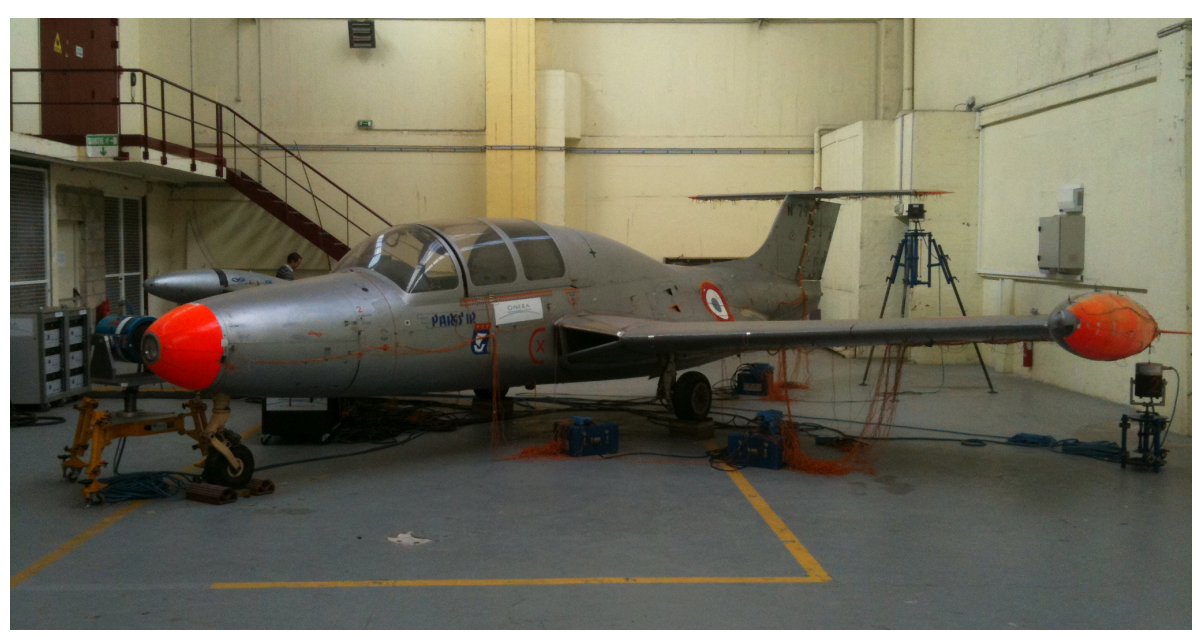

Figure 1: Morane-Saulnier Paris aircraft.

A total of 38 accelerometers is employed to instrument the structure, of which 8 tri-axial sensors are positioned at the two ends of each of the 4 wing-tank bolted attachments (Figure 2), where, thus, nonlinearities are mainly expected to be located. Two small-size shakers, one visible in Figure 1, are used to apply pure broad-band random excitation inputs to the two wings, along the vertical direction. The adopted sampling frequency is equal to $400 \mathrm{~Hz}$. Acceleration and force signals are both recorded at the driving points, located below the fuel tanks (Figure 3), by means of impedance heads.

In this paper, all the output channels related to bolted attachments are denoted by a label of the form abc:D, where a is Ri or Le, for indicating the right or the left side of the aircraft, respectively, $b$ is Fr or Re, when referring to the front or the rear bolted 
connection, and $\mathrm{c}$ is $\mathrm{Wi}$ or Ta, if the considered node belongs to the wing or to the tank, respectively. More over, D represents the channel direction (X, Y, or Z), evaluated with respect to a fixed reference frame, having the $\mathrm{X}$-axis coinciding with the longitudinal axis of the aircraft, but directed from the nose to the tail, the vertical, Z-axis, pointing upward, and the Y-axis chosen to form a right-handed cartesian coordinate system. Both the ends of the bolted connections are included when the character $c$ is imposed equal to BC. To give an example of this nomenclature, the label LeReWi:Y denotes the output channel referred to the left rear bolted connection, node on the wing, along the $Y$ direction. In the case of input channels, instead, we need only to distinguish between the left (Leln:Z) and the right (Riln:Z) excitation.

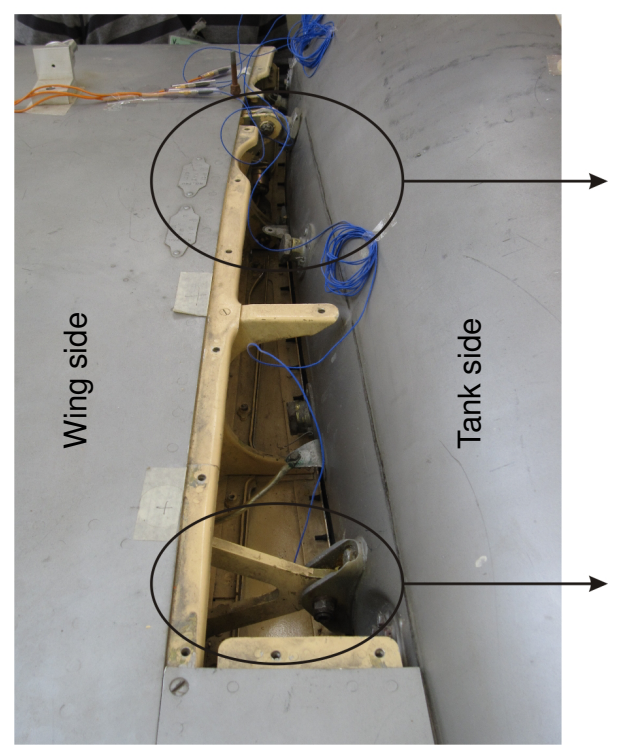

(a)

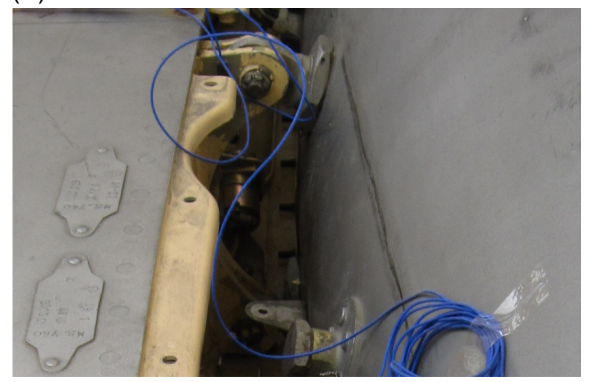

(b)

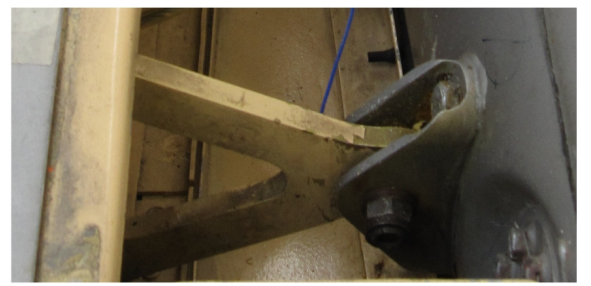

Figure 2: Connection between external fuel tank and wing tip (top view). Close-up of (a) front and (b) rear bolted attachments.

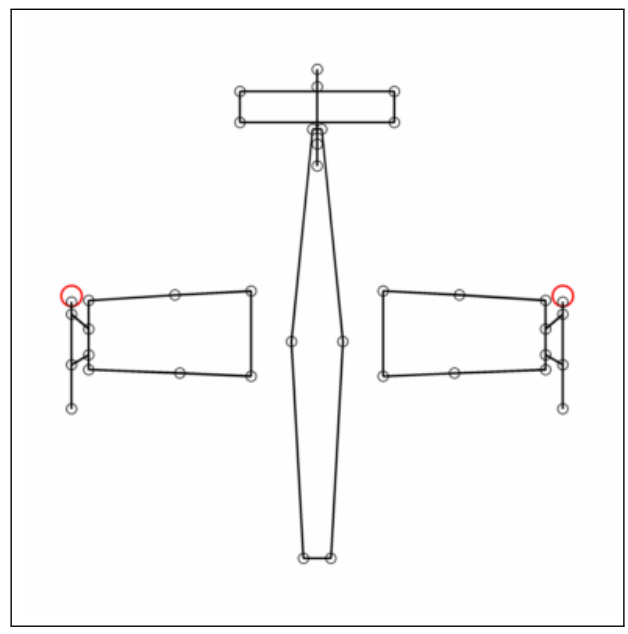

Figure 3: Layout of the experimental setup (top view). Black circles: output locations; red circles: driving points.

\section{NONLINEAR SUBSPACE IDENTIFICATION IN THE FREQUENCY DOMAIN}

The FNSI method derives models of mechanical systems possessing localised nonlinearities directly from measured data, and without resorting to a preexisting numerical model, e.g. a finite element model ${ }^{[6]}$. It is applicable to multi-input, multi-output structures with high nonproportional damping and high modal density, and makes no assumption as to the importance of nonlinearity in the measured dynamics ${ }^{[8]}$. The vibrations of such nonlinear systems are governed by Newton's second law

$$
\mathbf{M} \ddot{\mathbf{q}}(t)+\mathbf{C} \dot{\mathbf{q}}(t)+\mathbf{K} \mathbf{q}(t)+\mathbf{g}(\mathbf{q}(t), \dot{\mathbf{q}}(t))=\mathbf{p}(t)
$$


where $\mathbf{M}, \mathbf{C}, \mathbf{K} \in \mathbb{R}^{n \times n}$ are the mass, linear viscous damping and linear stiffness matrices, respectively; $\mathbf{q}(t)$ and $\mathbf{p}(t) \in \mathbb{R}^{n}$ are the generalized displacement and external force vectors, respectively; $\mathbf{g}(t) \in \mathbb{R}^{n}$ is the essentially nonlinear, i.e. nonlinearizable, restoring force vector encompassing elastic and dissipative contributions, and $n$ is the number of degrees of freedom (DOFs) of the structure obtained after spatial discretization. The amplitude, direction, location and frequency content of the excitation $\mathbf{p}(t)$ determine in which regime, linear or nonlinear, the structure behaves. The effects of the $r$ lumped nonlinear components in the system are represented using a linear-in-the-parameters model of the form

$$
\mathbf{g}(\mathbf{q}(t), \dot{\mathbf{q}}(t))=\sum_{a=1}^{r} \sum_{b=1}^{s_{a}} c_{a, b} \mathbf{h}_{a, b}(\mathbf{q}(t), \dot{\mathbf{q}}(t)) .
$$

In this double sum, $s_{a}$ is the number of nonlinear basis functions $\mathbf{h}_{a, b}(\mathbf{q}(t), \dot{\mathbf{q}}(t))$ selected to describe the $a$-th nonlinearity, and $c_{a, b}$ are the associated coefficients. The total number of nonlinear basis functions introduced in the model is equal to $s=\sum_{a=1}^{r} s_{a}$. Linearity in the parameters avoids an iterative optimization process, and issues related to initialization and convergence thereof.

Given measurements of $\mathbf{p}(t)$ and $\mathbf{q}(t)$ or its derivatives, and an appropriate selection of the functionals $\mathbf{h}_{a, b}(t)$, the FNSI algorithm aims at computing estimates of (i) the FRF matrix

$$
\mathbf{H}(\omega)=\left(-\omega^{2} \mathbf{M}+\mathbf{j} \omega \mathbf{C}+\mathbf{K}\right)^{-1}
$$

describing the underlying linear properties of the system in Eq. 1, where $\omega$ is the frequency and $\mathrm{j}$ the imaginary unit, and (ii) the nonlinear coefficients $c_{a, b}$ in Eq. 2.

The identification methodology essentially builds on a block-oriented interpretation of nonlinear structural dynamics, which sees nonlinearities as a feedback into the linear system in the forward loop ${ }^{[9]}$, as illustrated in Fig. 4. This interpretation boils down to moving the nonlinear internal forces in Eq. 1 to the right-hand side, and viewing them as additional external forces applied to the underlying linear structure, that is

$$
\mathbf{M} \ddot{\mathbf{q}}(t)+\mathbf{C} \dot{\mathbf{q}}(t)+\mathbf{K} \mathbf{q}(t)=\mathbf{p}(t)-\sum_{a=1}^{r} \sum_{b=1}^{s_{a}} c_{a, b} \mathbf{h}_{a, b}(\mathbf{q}(t), \dot{\mathbf{q}}(t)) .
$$

Based on Equation 4, the parameter estimation problem is solved in the frequency domain by processing a user-selected number

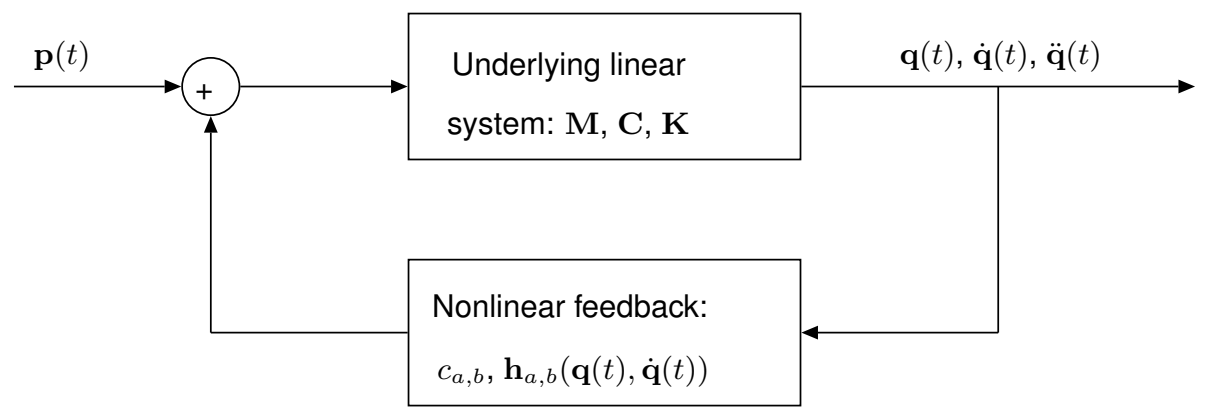

Figure 4: Feedback interpretation of nonlinear structural dynamics ${ }^{[9]}$.

of frequency lines in the measured band. A state-space representation of Eq. 4 is first extracted directly from frequency-domain data using robust tools from numerical analysis, including QR and singular value decompositions. The calculated state-space parameters are secondly converted into the modal space to estimate the underlying linear FRF matrix $\mathbf{H}(\omega)$, and to the physical space to estimate the nonlinear coefficients $c_{a, b}$. Fig. 5 presents an overview of the identification methodology. The interested reader is referred to Ref. ${ }^{[6]}$ for a detailed introduction to the theoretical and practical aspects of the FNSI method.

\section{LINEAR ANALYSIS AT LOW LEVEL AND NONLINEARITY DETECTION}

When the system is excited with low amplitude level inputs, the nonlinearities affecting the bolted connections are not activated. This means that data acquired at low level can be utilized to perform a linear identification of the structure. To this aim, a band- 
1. Select appropriate basis functions $\mathbf{h}_{a, b}(\mathbf{q}(t), \dot{\mathbf{q}}(t))$ to represent the nonlinearities.

2. Choose the number of processed frequency lines in the measured band.

3. Determine the order of the state-space representation of Eq. 4 and calculate its parameters from frequency-domain data.

4. Convert the state-space model to modal space to estimate the underlying linear FRF matrix $\mathbf{H}(\omega)$.

5. Convert the state-space model to physical space to estimate the nonlinear coefficients $c_{a, b}$.

Figure 5: Overview of the FNSI methodology.

limited $(7-45 \mathrm{~Hz})$ white-noise signal is applied to the aircraft, having a root-mean-square (RMS) amplitude of $4.4 \mathrm{~N}$. In this conditions, the FNSI method can be employed for obtaining the linear modal parameters of the structure. Since we, here, focus only on the identification of the first 5 modes, the frequency band of analysis ranging from 7 to $21.5 \mathrm{~Hz}$ is considered. A detailed motivation of this choice is reported in subsection 5.2 .

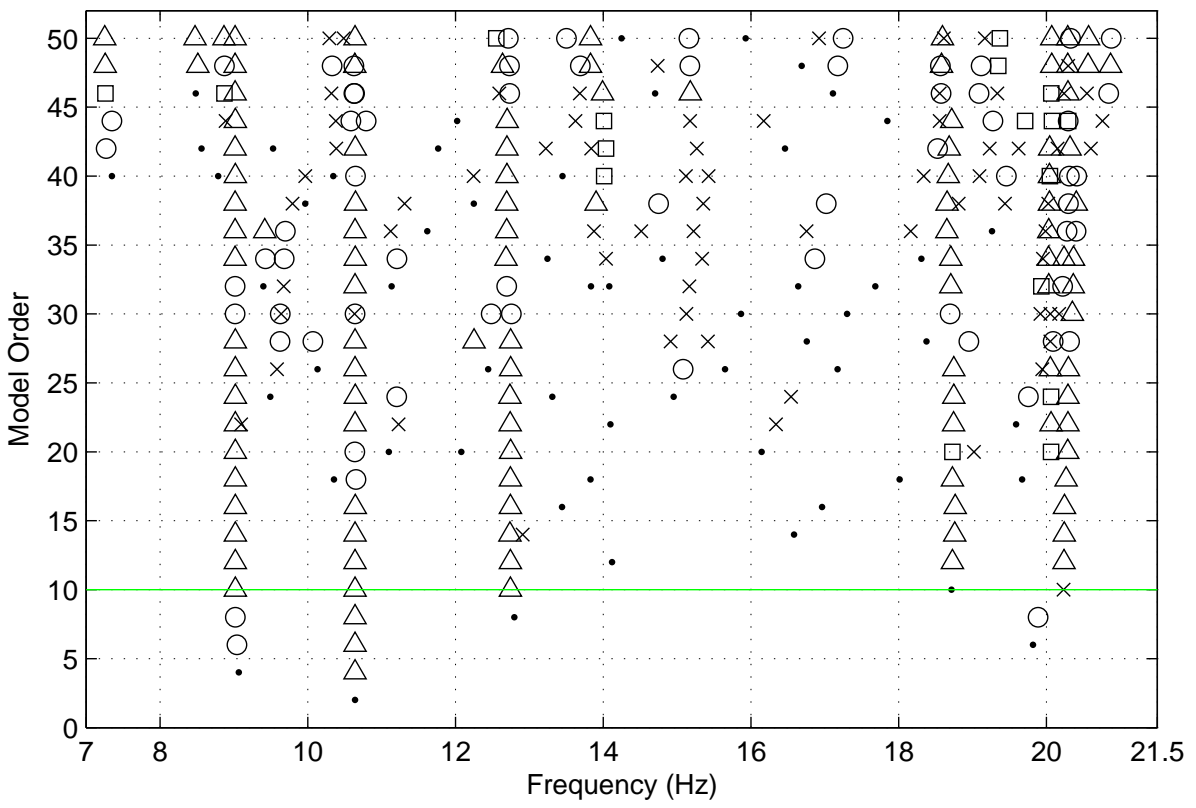

Figure 6: Stabilization diagram computed by the FNSI method at low level (4.4 N RMS). Dot: no stabilization in natural frequency; cross: stabilization in natural frequency; square: extra stabilization in damping ratio; circle: extra stabilization in MAC value; triangle: full stabilization. Stabilization thresholds for natural frequency, damping ratio and MAC value are $2 \%, 10 \%$ and 0.95 , respectively. The green line indicates the selected model order.

Figure 6 shows the linear properties extracted by the FNSI method for model orders up to 50 . This stabilization diagram leads to the identification of 5 mode shapes, whose natural frequencies and damping ratios are listed in Table 1. The obtained results are therein compared with those estimated by using another state-of-the-art algorithm, i.e. the poly-reference Least-Squares Complex Frequency Domain ( $p$-LSCFD ${ }^{[10]}$ ). Furthermore, in Figure 7 the comparison of results is performed by means of the modal assurance criterion (MAC ${ }^{[11]}$ ). By considering what is shown we may conclude that the two methods give basically the same results, both in terms of natural frequencies and mode shapes, while a bias error inevitably affects the estimate of the damping ratios. However, a good agreement is found when comparing FRFs synthesized by the FNSI method and those obtained with the $\mathrm{H} 1$ estimator (Figure 8).

A second random data set measured at high level (44 N RMS) is then analyzed to detect and locate in the frequency domain 
(a)



(b)

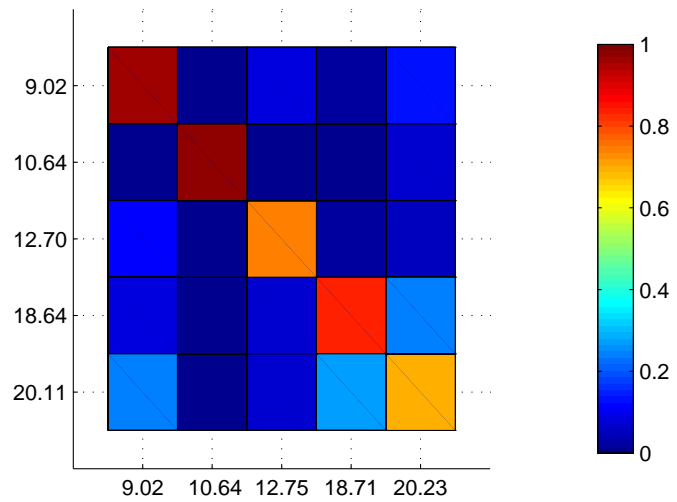

Figure 7: Comparison between FNSI and p-LSCFD results. (a) AutoMAC for FNSI method; (b) MAC between FNSI and p-LSCFD mode sets.
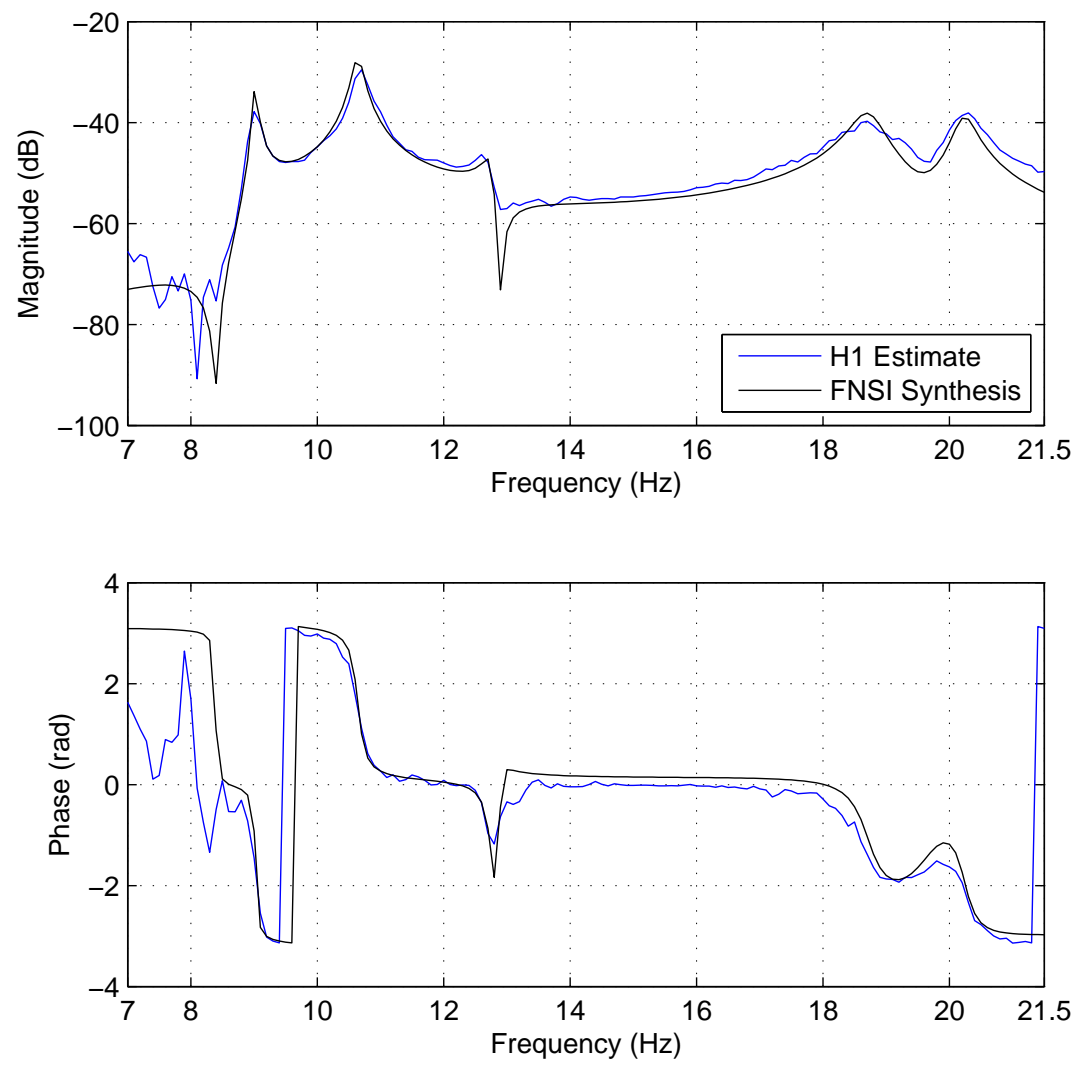

Figure 8: FRF LeReWi:Y/Riln:Z. Comparison between H1 estimate and FNSI synthesis at low level (4.4 N RMS). 


\begin{tabular}{|c|c|c|c|c|c|c|}
\cline { 2 - 7 } \multicolumn{1}{c|}{} & \multicolumn{2}{c|}{ FNSI } & \multicolumn{2}{c|}{ p-LSCFD } & \multicolumn{2}{c|}{ Discrepancy } \\
\hline \hline Mode & $f_{n}(\mathrm{~Hz})$ & $\zeta(\%)$ & $f_{n}(\mathrm{~Hz})$ & $\zeta(\%)$ & $\delta_{f}(\%)$ & $\delta_{\zeta}(\%)$ \\
\hline \hline 1 & 9.02 & 0.29 & 9.02 & 0.32 & 0.00 & 9.38 \\
\hline 2 & 10.64 & 0.78 & 10.64 & 0.78 & 0.00 & 0.00 \\
\hline 3 & 12.75 & 0.67 & 12.70 & 0.56 & 0.39 & 19.64 \\
\hline 4 & 18.71 & 1.34 & 18.64 & 2.10 & 0.38 & 36.19 \\
\hline 5 & 20.23 & 0.86 & 20.11 & 0.84 & 0.60 & 2.38 \\
\hline
\end{tabular}

TABLE 1: Comparison between FNSI and p-LSCFD results. Estimated natural frequencies $\left(f_{n}\right)$ and damping ratios $(\zeta)$ in the 7 $-21.5 \mathrm{~Hz}$ band.

the modes triggering nonlinear mechanisms. Since all the considered mode shapes involve to a certain extent the wings' deformation, all the frequencies seem to be affected by nonlinear distortions. To give an example, the deformed shapes of modes 2 and 4 are depicted in Figures 9a and 9b, respectively.

(a)

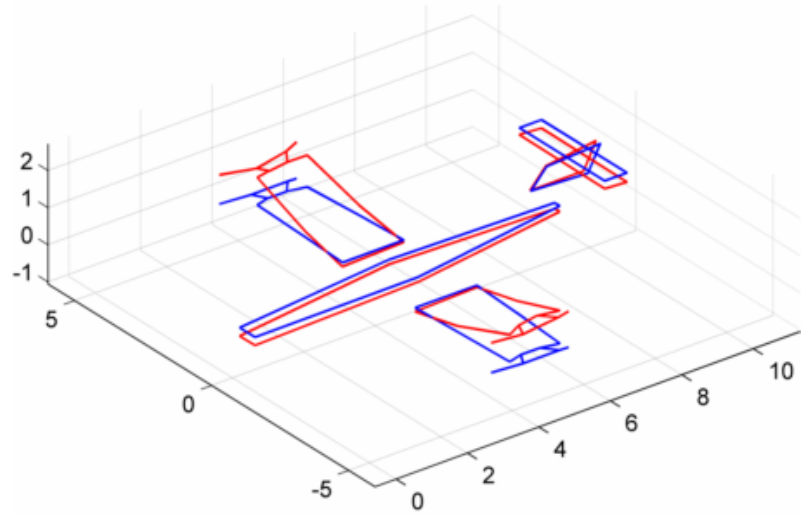

(b)

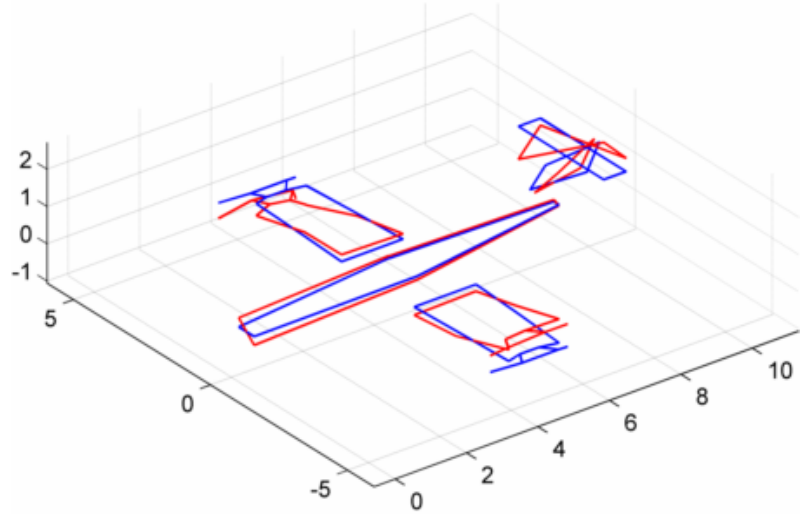

Figure 9: Deformed shapes corresponding to modes 2 and 4 identified at low level (4.4 N RMS). Geometrical dimensions are given in meters. (a) Mode $2(10.64 \mathrm{~Hz})$ and (b) mode $4(18.71 \mathrm{~Hz})$. Blue and red lines indicate the undeformed and deformed shapes, respectively.

In Figure 10, the comparison between the low- and high-level FRFs is presented. A softening behavior is clearly visible for all the first 3 modes, included in the range from 9 to $13 \mathrm{~Hz}$. Modes 4 and 5 represent the most critical configurations in terms of deformed shapes. During the resonance, in fact, the bolted attachments are activated through a combination of wing bending and torsion. As a consequence, the related modal parameters are strongly affected by nonlinear distortions. Since the peak located at $18.7 \mathrm{~Hz}$ is more depressed at high level, the interpretation of the nonlinear behavior becomes arduous. However, by looking at the phase plot, one may conclude that the noticeable curve translation is still induced by softening effect. We instead demonstrate in what follows, that this phenomenon can likely to be attributed to loosening in the bolted connections. In fact, even if at high level an overall softening effect has been detected, we later show that some connections can exhibit a combined softening-hardening behavior.

\section{NONLINEARITY IDENTIFICATION AT HIGH LEVEL}

To obtain a nonlinear subspace model of the aircraft at the high $44 \mathrm{~N} \mathrm{RMS} \mathrm{level,} \mathrm{a} \mathrm{five-step} \mathrm{procedure} \mathrm{is} \mathrm{followed} \mathrm{(see} \mathrm{Figure} \mathrm{5).}$ The first step is an appropriate selection of the nonlinear basis functions $\mathbf{h}_{a, b}(\mathbf{q}(t), \dot{\mathbf{q}}(t))$ defined in Eq. 2. The analysis reported in Section 4 has revealed that the physical phenomena activating the nonlinear behavior of the structure are complex and numerous. Since the aircraft is equipped with 4 bolted attachments and the acceleration is measured at the two ends along each direction, 24 output channels are candidate to form the right basis functions. In the following section, two useful tools are introduced: i) cubic splines, that avoid the selection of physics-based functional forms; ii) the comparison approach, that allows for evaluating the influence of the selected basis functions on the retrieved underlying linear properties. 

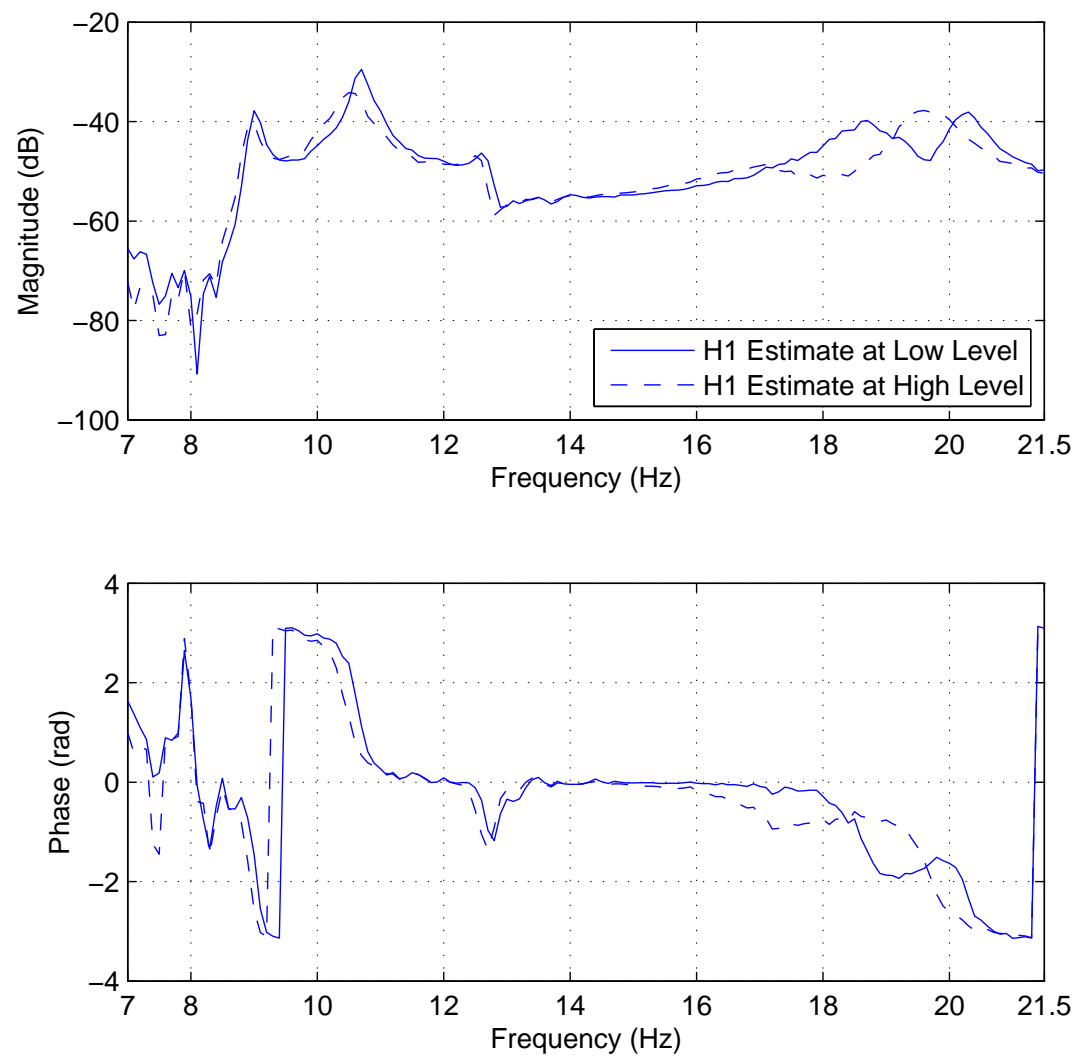

Figure 10: FRF LeReWi:Y/Riln:Z. Comparison between H1 estimate at low (4.4 N RMS) and high (44 N RMS) levels.

\subsection{Selection of appropriate basis functions}

For the sake of simplicity and without loss of generality, the scope of this section is restricted to an elastic nonlinear restoring force $g(q(t))$ in the single-DOF case. Let $q$ be divided into $L$ segments of arbitrary length and defined by their abscissas, denoted by $q_{k}$ for $k=1, \ldots, L+1$. Each abscissa is associated with an ordinate $g_{k}$, together defining a knot $\left(q_{k}, g_{k}\right)$ of the spline. Thus, if $q$ is a displacement value in between knots $k$ and $k+1$, the corresponding point of a cubic-spline-based approximation of $g(q(t))$ is given by ${ }^{[12]}$

$$
g(q(t))=\left(2 t^{3}-3 t^{2}+1\right) g_{k}+\left(-2 t^{3}+3 t^{2}\right) g_{k+1}+\left(t^{3}-2 t^{2}+t\right)\left(q_{k+1}-q_{k}\right) g_{k}^{\prime}+\left(t^{3}-t^{2}\right)\left(q_{k+1}-q_{k}\right) g_{k+1}^{\prime}
$$

where $t$ is the normalised displacement $\frac{q-q_{k}}{q_{k+1}-q_{k}}$. The computation of the first derivatives $g_{k}^{\prime}=\partial g_{k} / \partial q_{k}$ can be achieved by forcing the cubic spline and its first two derivatives to be continuous across each of the interior knots. This results in $L-1$ linear constraint equations

$$
\frac{g_{k-1}^{\prime}}{q_{k}-q_{k-1}}+2\left(\frac{1}{q_{k}-q_{k-1}}+\frac{1}{q_{k+1}-q_{k}}\right) g_{k}^{\prime}+\frac{g_{k+1}^{\prime}}{q_{k+1}-q_{k}}=3\left(\frac{g_{k}-g_{k-1}}{\left(q_{k}-q_{k-1}\right)^{2}}+\frac{g_{k+1}-g_{k}}{\left(q_{k+1}-q_{k}\right)^{2}}\right) .
$$

Since the essentially nonlinear restoring force $g(q(t))$ is zero and has zero slope at equilibrium, one should also enforce, in the segment containing the abscissa of the equilibrium point, that

$$
\left(t_{0}^{3}-2 t_{0}^{2}+t_{0}\right)\left(q_{k+1}-q_{k}\right) g_{k}^{\prime}+\left(t_{0}^{3}-t_{0}^{2}\right)\left(q_{k+1}-q_{k}\right) g_{k+1}^{\prime}=-\left(2 t_{0}^{3}-3 t_{0}^{2}+1\right) g_{k}-\left(-2 t_{0}^{3}+3 t_{0}^{2}\right) g_{k+1}
$$

and

$$
\left(3 t_{0}^{2}-4 t_{0}+1\right)\left(q_{k+1}-q_{k}\right) g_{k}^{\prime}+\left(3 t_{0}^{2}-2 t_{0}\right)\left(q_{k+1}-q_{k}\right) g_{k+1}^{\prime}=6\left(t_{0}-t_{0}^{2}\right)\left(g_{k}-g_{k+1}\right)
$$


where $t_{0}=\frac{-q_{k}}{q_{k+1}-q_{k}}$. Eqs. 6,7 and 8 constitute $L+1$ relations that uniquely define the $L+1$ parameters $g_{1}^{\prime}, \ldots, g_{L+1}^{\prime}$ as functions of the ordinates of the knots $g_{1}, \ldots, g_{L+1}$. The first derivatives can thus be substituted in Equation 5 to compute the basis functions associated with the ordinates. These basis functions correspond to the terms $\mathbf{h}_{a, b}(t)$ introduced as additional external forces in the FNSI algorithm, as described in Section 3.

The optimal number of knots in the definition of the splines should rigorously be sought by minimizing the difference, in some metric, between the predictions of the nonlinear model and measured data. In practice, an acceptable number of knots can be found by a trial and error approach, by maximizing the accuracy of the underlying linear properties of the system, estimated from nonlinear data. However, when a large number of outputs is employed to form the basis functions, the inverse problem becomes difficult to be solved. This is mainly due to the high dimensionality of data matrices involved in the computation. This means that the maximum allowed number of basis functions is inevitably influenced by the size of the data set utilized in the identification.

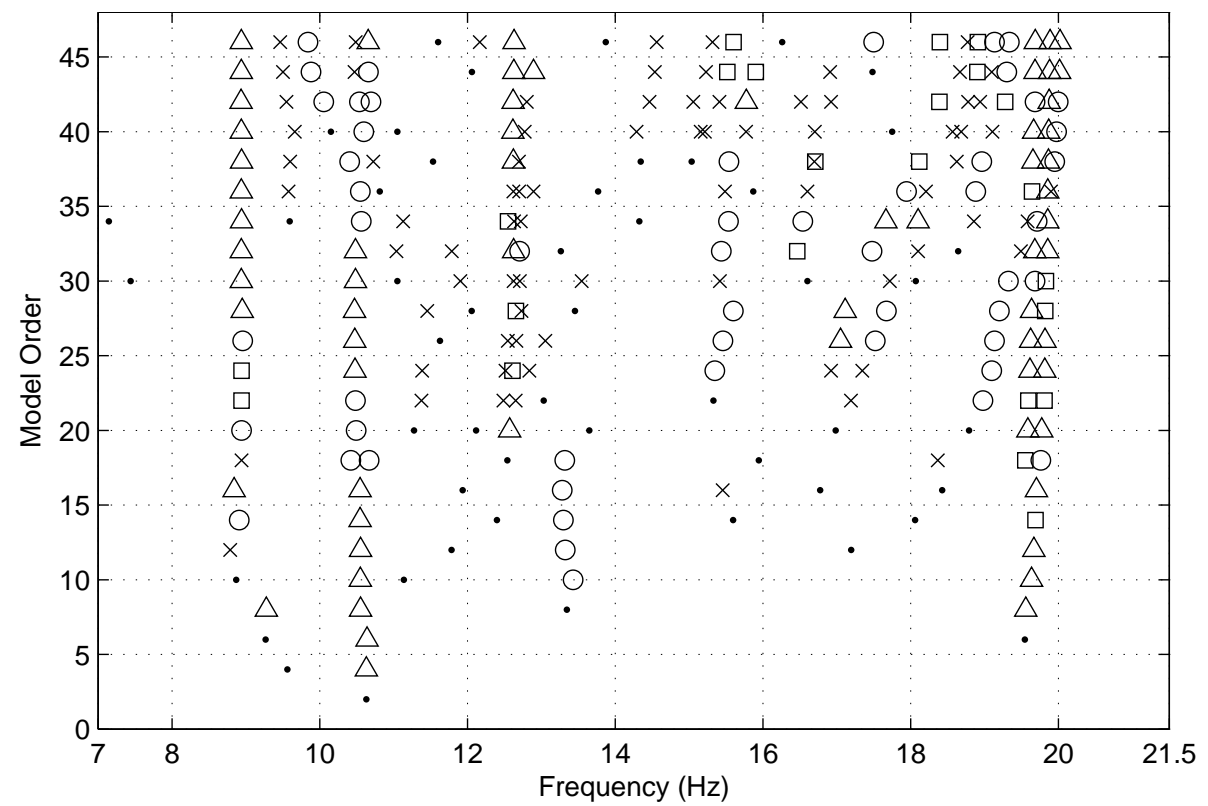

Figure 11: Stabilization diagram computed by the FNSI method at high level (44 N RMS). Dot, crosses, squares, circles and triangles are defined similarly to Fig. 6.

In this framework, the comparison approach represents a novel strategy, developed to solve the above described problems. The main idea is to single out the minimum number of outputs and the simplest functional forms, which are able to retrieve at high level enough accurate underlying linear properties. The first step is to collect natural frequencies, damping ratios and real mode shapes estimated by the FNSI method at low level. Tolerance thresholds are imposed by the analyst to these three parameters. In particular, the tolerance on natural frequencies is utilized to form the acceptance regions. Of course, the number of these regions has to be equal to the number of the collected natural frequencies. Then, at high level, the outputs that are candidates to form the basis functions have to be combined in a series of scheduled experiments. The underlying linear properties are extracted for all the combinations and for each model order. The obtained solutions are stacked in the acceptance regions and the discarded linear properties are immediately classified as non comparable in natural frequency. At each model order, at least one admitted solution must be found in all the acceptance regions. An ultimate step is needed to reveal the so called comparable model orders, in which the underlying linear properties are retrieved within certain tolerance limits. A graphical tool, that we name comparison diagram, is developed to make simple and effective the visualization of the comparable model orders and of equivalent solutions. On the x-axis, the natural frequencies are represented, while the model orders are given on the $y$-axis; horizontal green lines indicate the comparable model orders. As in the case of stabilization diagram, several situations are labeled with different symbols: The dot denotes no equivalence in natural frequency, the cross represents equivalence in natural frequency, the square and the circle stand for extra equivalence in damping ratio and in MAC value, the triangle refers to fully equivalent solutions.

For the investigated aircraft, the modal parameters estimated at low level are collected in Table 1. Comparison thresholds for natural frequency, damping ratio and MAC value are set to $1.25 \%, 10 \%$ and 0.95 , respectively. The motivation of these choices is later discussed in Subsection 5.4. We choose to include in each experiment, only one direction for each bolted connection. 


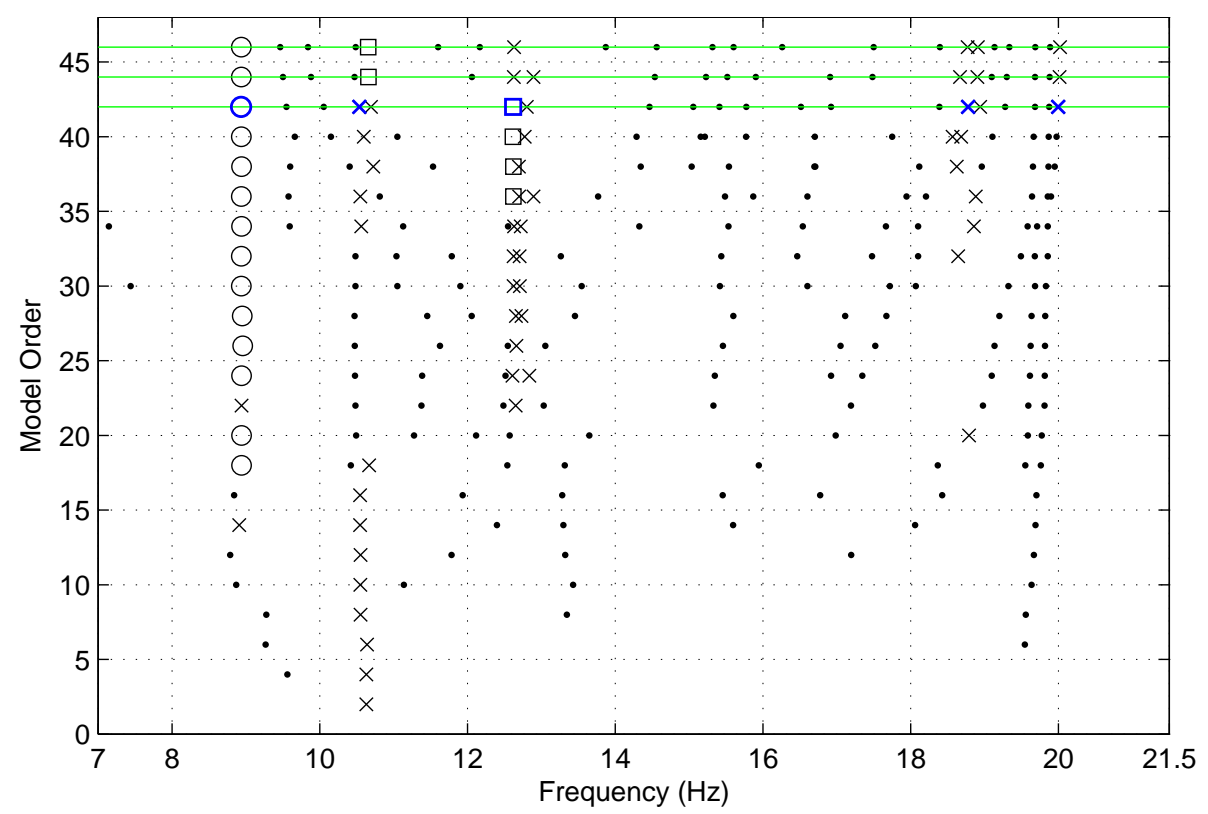

Figure 12: Comparison diagram computed at high level (44 N RMS). Splines with 5 knots on relative displacements of the outputs denoted as RiReBC:Z, RiFrBC:Z, LeReBC:Y and LeFrBC:X are included in the FNSI routine. Dot: no equivalence in natural frequency; cross: equivalence in natural frequency; square: extra equivalence in damping ratio; circle: extra equivalence in MAC value; triangle: full equivalence. Comparison thresholds for natural frequency, damping ratio and MAC value are $1.25 \%$, $10 \%$ and 0.95 , respectively. The green lines are the comparable model orders and the blue marks indicate the selected system poles.

Thus, employing a full factorial Design of Experiments with 4 factors (the 4 attachments) and 3 levels (the 3 directions), a table of 81 possible combinations is obtained. With this strategy, only 8 of the 24 output channels related to the bolted connections have to be included in the FNSI routine. By the comparison approach, it is found that splines with 5 knots on relative displacements are the simplest functional forms able to suitably capture the physical mechanisms activating the nonlinear phenomena. It is worth to here stress the reduced dimensionality of the consequent inverse problem, that encompasses 20 basis functions, 2 external forces, 32 outputs for the aircraft main body and 8 outputs for the bolted connections.

\subsection{Choice of the processed bandwidth}

As anticipated in Section 4, the bandwidth processed for the identification ranges from 7 to $21.5 \mathrm{~Hz}$. This choice is principally made for reducing the computational burden. In fact, the dimensionality reduction operated through the comparison approach may become not sufficient when the FNSI method has to deal with a considerable number of basis functions. The restriction of the bandwidth is helpful for avoiding memory issues, even if does not allow for preserving the nonlinear distortions usually appearing outside the whole band of excitation (from 7 to $45 \mathrm{~Hz}$ ). Owing to this dimensionality, time-domain identification techniques, as, in particular, the subspace method proposed in Ref. ${ }^{[5]}$, are likely to suffer from computational memory issues, because of their inability to reject less informative measured samples. Moreover, few methods in the technical literature are capable of reliably handling a large amount of nonlinear coefficients. For instance,the conditioned reverse path method Ref. ${ }^{[13]}$ estimates the coefficients sequentially, i.e. errors are accumulated throughout the identification process, and would probably lead to very large errors in the present case study. Another example is the nonlinear identification through feedback of the outputs method Ref. ${ }^{[9]}$, whose estimation accuracy is known to decrease rapidly as the number of parameters increases.

\subsection{Determination of the model order}

The third step in Figure 5 is the determination of the model order. One can notice that, here, the information about the poles' stabilization may become ambiguous and difficult to be interpreted (Fig. 11). In modal analysis, the stabilization diagram is often used to assist the analyst in separating the physical system poles from the mathematical ones. Since the linear identification 
has been accomplished at low level, the correct physical poles have been already collected. This means that in the comparison diagram the spurious poles are a priori discarded, since they are classified as non comparable in natural frequency at the beginning of the analysis. On the contrary, the stabilization diagram may lead to the selection of stable, but not comparable underlying linear properties. These considerations remark the importance of the proposed strategy, in which the comparison approach represents a useful methodology that allows for: i) refining the localization of nonlinearities, and ii) revealing the presence of comparable model orders.
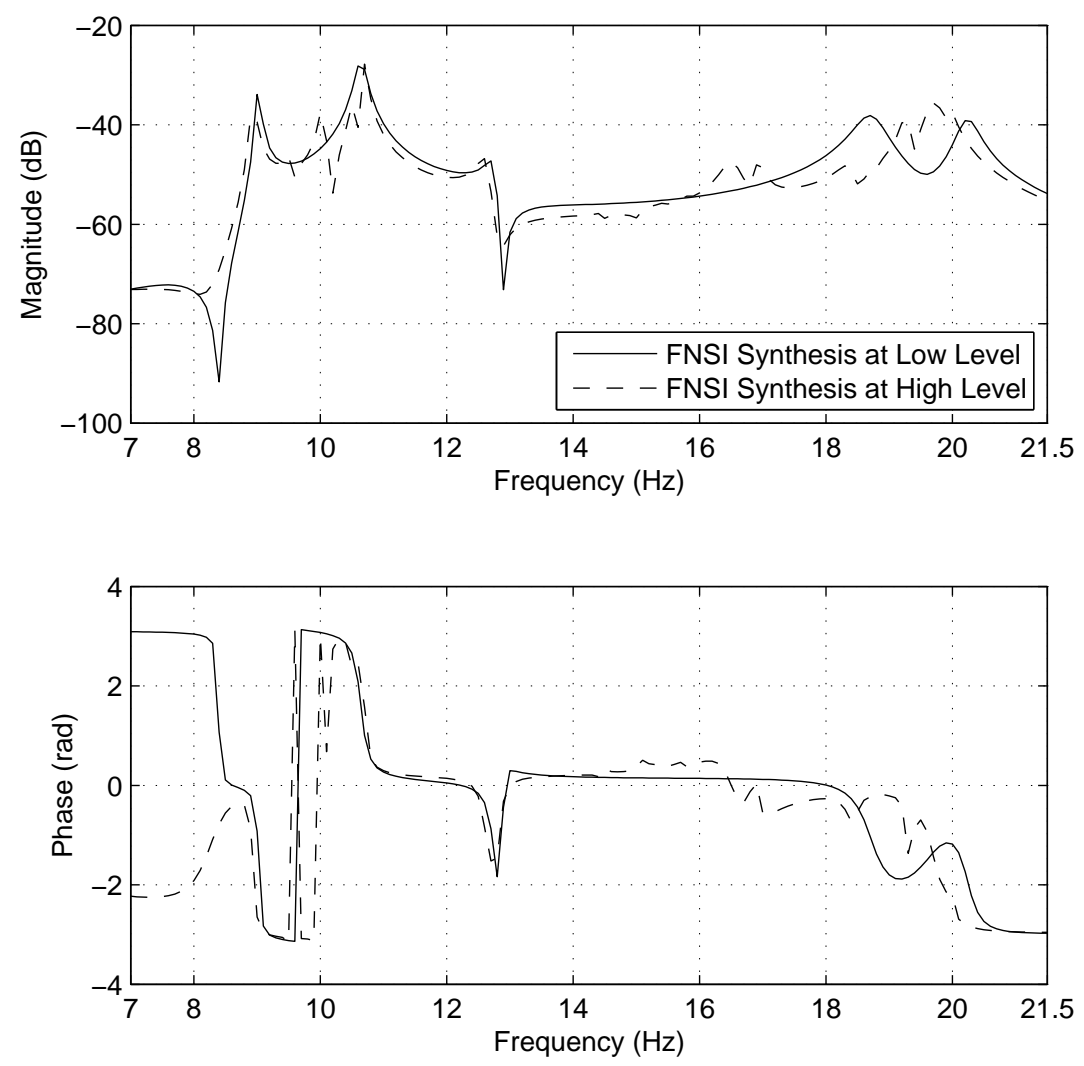

Figure 13: FRF LeReWi:Y/Riln:Z. Comparison between FNSI synthesis at low (4.4 N RMS) and high (44 N RMS) levels.

\subsection{Estimation of the underlying linear properties}

In Figure 12 we show the comparison diagram corresponding to the experiment that involves the outputs denoted as RiReBC:Z, RiFrBC:Z, LeReBC:Y and LeFrBC:X. Three consecutive model orders, i.e. the 42, 44 and 46, are found to be comparable. An extra equivalence in the MAC value is evident only for the first mode. Depending on the chosen model order, the damping ratio may result comparable on the third or on the second mode. Of course, these outcomes are absolutely dependent on the imposed tolerance limits. We stress that the comparison approach is really sensitive to tolerance fixed on the natural frequencies, mainly owing to the construction of the acceptance regions. Obviously, the larger are these regions, the higher is the chance of finding comparable model orders. On the opposite, using strict tolerance values leads to the estimate of more accurate underlying linear properties. Moving from these considerations, the analyst is suggested to set the comparison thresholds according to the application. In Figure 10 a small shift can be noticed for the first 3 peaks of the FRF estimated at high level. This means that, for the present investigation, the adoption of severe tolerance limits is strongly recommended.

The FRF LeReWi:Y/Riln:Z synthesized by the FNSI at model order 42 is depicted in Figure 13. When compared to the synthesis obtained at low level, many additional peaks are now visible, which corresponds to the numerous spurious poles present at this model order. Due to the extra equivalence on the damping ratio, a good agreement is observable only at the third resonant 
frequency $(12.75 \mathrm{~Hz})$. A useful way to improve this result is presented in Section 6.

\subsection{Estimation of the nonlinear coefficients}

With the last step in Figure 5, the state-space model is converted to the physical space, in order to visualize the restoring forces, fitted by using cubic splines. To this end, the conversion scheme detailed in Ref. ${ }^{[6]}$ is utilized. As a result, the nonlinear coefficients identified from the extended FRF matrix $\mathbf{H}_{e}(\omega)$ are spectral quantities, i.e. are complex-valued and frequencydependent. A reliable identification, together with an appropriate selection of the basis functions, should make the imaginary parts much smaller than the corresponding real parts. The coefficients' frequency dependence should also remain small. These indications are usually utilized as criteria for the quality assessment of the estimated nonlinear parameters. In Figure 14, we illustrate the real and the imaginary parts of the complex-valued coefficients related to the output LeFrBC:X, whose estimation results to be that more affected by errors. The variations along the frequency axis are located at the same positions of the selected modal parameters. As one can clearly observe, on both the curves the spurious poles give rise to sharpest peaks, in turn, heavily affecting the average values of the estimated coefficients. To mitigate this effect, the frequency range for the conversion is restricted to the flat zone ranging from 13 to $14 \mathrm{~Hz}$ (Fig. 14). In this range, the ratios between the averaged real and imaginary parts are computed, actually revealing the output LeFrBC:X as the worst estimated among the four of the experiment (see the fourth row of Table 2). This result is even improved in the next section (Sec. 6), by using an appropriate strategy specifically designed for removing the spurious poles.

(a)

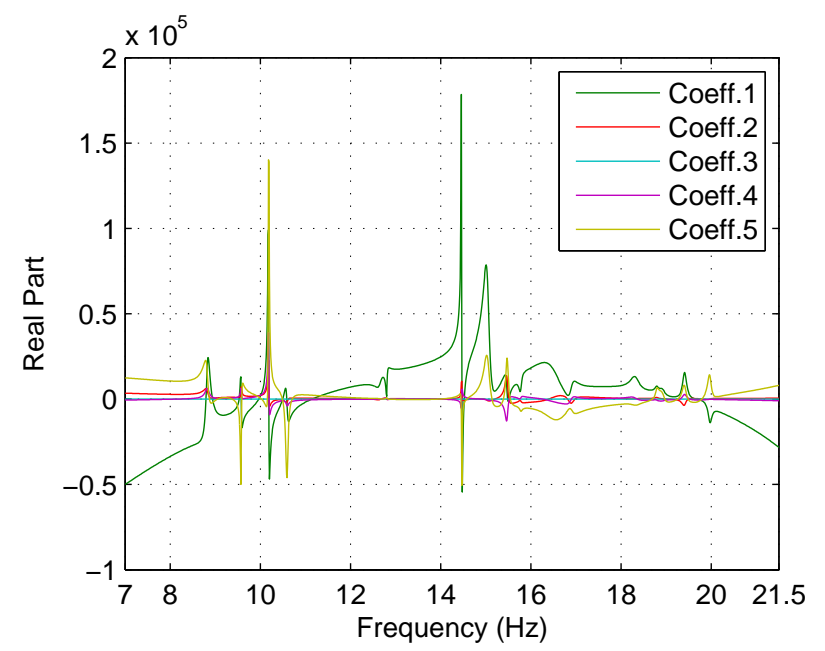

(b)

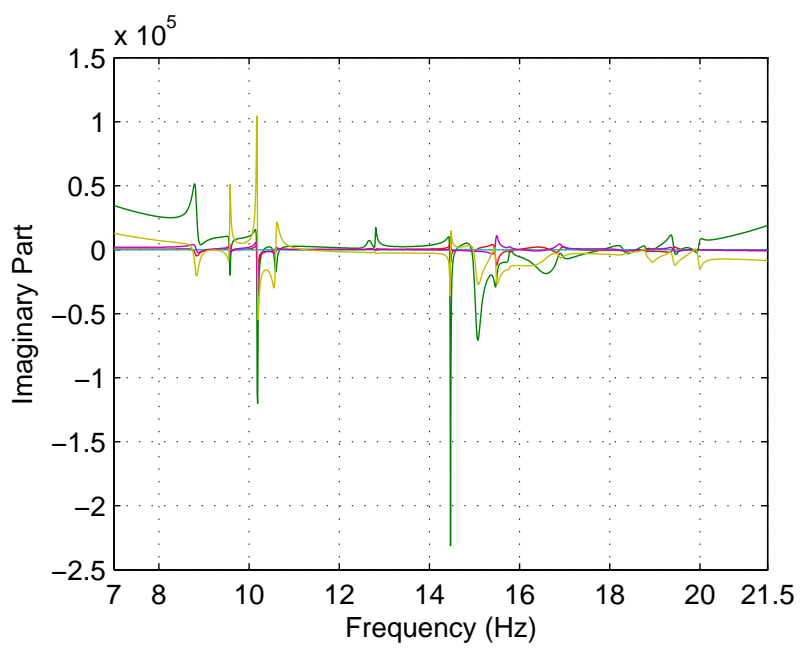

Figure 14: Complex-valued estimates of the 5 nonlinear coefficients related to the output LeFrBC:X in the full bandwidth (7 $21.5 \mathrm{~Hz})$.

\begin{tabular}{|c|c|c|c|c|c|}
\hline Location & Coeff. 1 & Coeff. 2 & Coeff. 3 & Coeff. 4 & Coeff. 5 \\
\hline \hline RiReBC:Z & 0.16 & -0.10 & 0.92 & 0.33 & 0.08 \\
\hline RiFrBC:Z & 1.84 & 0.80 & 1.38 & 1.33 & 1.02 \\
\hline LeReBC:Y & 0.16 & -0.90 & 1.70 & -0.42 & 0.98 \\
\hline LeFrBC:X & 0.87 & -0.38 & 0.02 & -0.71 & -1.10 \\
\hline
\end{tabular}

TABLE 2: Ratio between averaged real and imaginary parts in logarithmic scaling. The estimate is performed in the $13-14 \mathrm{~Hz}$ band.

\section{PROPOSED STRATEGY FOR REMOVING SPURIOUS POLES AND IMPROVED RESULTS}

The presence of the spurious poles can be attributed to many different causes, e.g. noise on the data, modeling errors, computational issues, etc. Classical subspace methods do not provide the user with the possibility of selecting poles computed at a given model order, not allowing for discarding the spurious ones. In the usual procedure, in fact, all the poles have to be included. 
To avoid this drawback, an appropriate novel strategy is employed for removing the spurious poles. In the first part of the FNSI method, the underlying linear properties are directly computed from the continuous state and output matrices $\mathbf{A}_{c} \in \mathbb{R}^{2 n \times 2 n}$ and $\mathbf{C} \in \mathbb{R}^{l \times 2 n}$. Then, the discrete extended input and direct feedthrough matrices $\mathbf{B}_{d}^{e} \in \mathbb{R}^{2 n \times(m+s)}$ and $\mathbf{D}^{e} \in \mathbb{R}^{l \times(m+s)}$ are calculated by defining the transfer function matrix $\mathbf{G}_{s}(k)$ associated with the state-space model

$$
\mathbf{G}_{s}(k)=\mathbf{C}\left(z_{k} \mathbf{I}^{n \times n}-\mathbf{A}_{d}\right)^{-1} \mathbf{B}_{d}^{e}+\mathbf{D}^{e}
$$

and minimizing the weighted difference between the measured and modeled output spectra in a linear least-squares sense

$$
\mathbf{B}_{d}^{e}, \mathbf{D}^{e}=\arg \min \sum_{k=1}^{F} \mathbf{W}^{2}(k)\left|\mathbf{Y}(k)-\mathbf{H}_{e}(k) \mathbf{E}(k)\right|^{2}
$$

where $z_{k}=\mathrm{e}^{\mathrm{j} 2 \pi k / \mathrm{N}}$ is the Z-transform variable for discrete-time models, $\mathbf{W}(k)$ is a real-valued weighting function, $\mathbf{Y}(k)$ and $\mathbf{E}(k)$ represent the output spectra and the extended input vector, $F$ denotes the number of frequency samples, the subscripts $c$ and $d$ refer to continuous and discrete time models, while $l$ and $m$ indicate the number of outputs and the number of inputs involved in the identification, respectively. The continuous-time state-space matrices can be converted in discrete-time form by means of the following relations, considering a sampling frequency $f_{s}$

$$
\mathbf{A}_{d}=\operatorname{expm}\left(\mathbf{A}_{c} / f_{s}\right) \quad \mathbf{B}_{d}^{e}=\left(\operatorname{expm}\left(\mathbf{A}_{c} / f_{s}\right)-\mathbf{I}^{2 n \times 2 n}\right) \mathbf{A}_{c}^{-1} \mathbf{B}_{c}^{e}
$$

where expm indicates the matrix exponential. The employed procedure for the removal of spurious poles consists in the reduction of the dimensionality of the state-space matrices. From the eigenvalue decomposition of $\mathbf{A}_{c}$ the poles of the system and the eigenvectors are collected in $\boldsymbol{\Lambda}_{c} \in \mathbb{C}^{2 n \times 2 n}$ and $\mathbf{V} \in \mathbb{C}^{2 n \times 2 n}$, respectively. Once $n_{p}$ poles are selected by the analyst, the corresponding diagonal terms in $\boldsymbol{\Lambda}_{c}$ are extracted and a reduced matrix of system poles is obtained $\overline{\boldsymbol{\Lambda}}_{c} \in \mathbb{C}^{2 n_{r} \times 2 n_{r}}\left(n_{r}=2 \cdot n_{p}\right)$. The same procedure of extraction is repeated on the columns of $\mathbf{V}$, leading to $\overline{\mathbf{V}} \in \mathbb{C}^{2 n \times 2 n_{r}}$. The reduced output matrix $\overline{\mathbf{C}} \in \mathbb{C}^{l \times 2 n_{r}}$ is, thus, calculated in modal coordinates

$$
\overline{\mathbf{C}}=\mathbf{C} \overline{\mathbf{V}}
$$

and the following Eq. 13 is utilized to replace Eq. 9 in the minimization problem, in order to derive the $\overline{\mathbf{B}}_{d}^{e}$ and $\mathbf{D}^{e}$ matrices

$$
\mathbf{G}_{s}(k)=\overline{\mathbf{C}}\left(z_{k} \mathbf{I}^{n \times n}-\overline{\mathbf{\Lambda}}_{d}\right)^{-1} \overline{\mathbf{B}}_{d}^{e}+\mathbf{D}^{e} .
$$

The array $\overline{\mathbf{B}}_{d}^{e} \in \mathbb{C}^{2 n_{r} \times(m+s)}$ is the discrete extended input matrix represented in modal coordinates. We comment that for converting $\bar{\Lambda}_{c}$ and $\overline{\mathbf{B}}_{d}^{e}$ to discrete and continuous time forms the same relations holding for $\mathbf{A}_{c}$ and $\mathbf{B}_{d}^{e}($ Eq. 11) can be employed, respectively. Finally, the extended FRF matrix $\mathbf{H}_{e}(\omega)$ is estimated and the typical scheme for conversion to the physical space can be applied to retrieve the nonlinear coefficients.

\begin{tabular}{|c|c|c|c|c|c|c|}
\cline { 2 - 7 } \multicolumn{1}{c|}{} & \multicolumn{2}{c|}{ FNSI at Low Level } & \multicolumn{2}{c|}{ FNSI at High Level } & \multicolumn{2}{c|}{ Error } \\
\hline \hline Mode & $f_{n}(\mathrm{~Hz})$ & $\zeta(\%)$ & $f_{n}(\mathrm{~Hz})$ & $\zeta(\%)$ & $\epsilon_{f}(\%)$ & $\epsilon_{\zeta}(\%)$ \\
\hline \hline 1 & 9.02 & 0.29 & 8.94 & 0.42 & 0.89 & 44.83 \\
\hline 2 & 10.64 & 0.78 & 10.54 & 0.50 & 0.94 & 35.90 \\
\hline 3 & 12.75 & 0.67 & 12.62 & 0.72 & 1.02 & 7.46 \\
\hline 4 & 18.71 & 1.34 & 18.78 & 0.16 & 0.37 & 88.06 \\
\hline 5 & 20.23 & 0.86 & 20.00 & 0.20 & 1.14 & 76.74 \\
\hline
\end{tabular}

TABLE 3: Comparison between FNSI estimates at low and high levels. Estimated natural frequencies $\left(f_{n}\right)$ and damping ratios $(\zeta)$ in the $7-21.5 \mathrm{~Hz}$ band. The fourth and the fifth columns refers to underlying linear properties selected in Fig. 12.

In the comparison diagram of Figure 12 the blue marks indicate the selected system poles at model order 42 . In Table 3 , the linear properties identified at low level (see Section 4), together with the associated frequencies estimated at high level are reported. As one can see, the error values are respectful of the imposed comparison thresholds and this means that, since strict tolerances can be fixed on natural frequencies, a consequent matching is found between estimates at low and high levels. Differently, the overall estimate of damping ratios is less satisfactory. The damping ratios that present higher discrepancies are those corresponding to modes 4 and 5. Probably, this fact may be related to the activation of nonlinear phenomena involving damping forces. This behavior, already singled out during the detection step, is further stressed and put in evidence by the comparison diagram. In fact, the last region ranging from 17 to $21 \mathrm{~Hz}$ is very confused and really affected by the presence of not comparable solutions. We even comment that the splines utilized in the present identification are not able to capture this kind of behavior, being solely defined on the relative displacement. Future investigations will be made in this direction. 

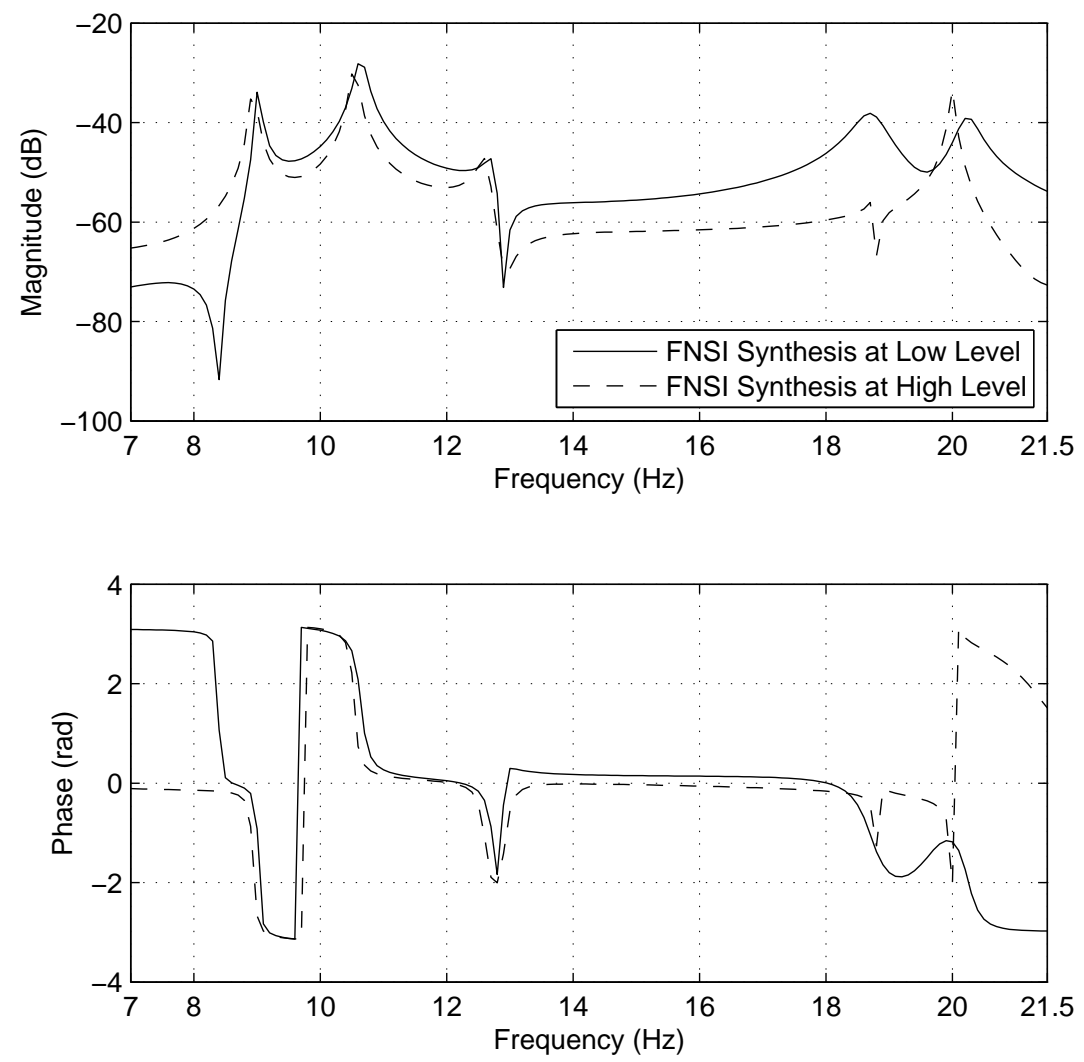

Figure 15: Improved FRF LeReWi:Y/Riln:Z. Comparison between FNSI synthesis at low (4.4 N RMS) and high (44 N RMS) levels. The selected poles are depicted in Fig. 12.

The results presented in Table 3 have a direct influence on the synthesis of FRFs. The same FRF described in the previous section (Sec. 5), i.e. the one of output LeReWi:Y/Riln:Z, is depicted in Figure 15, where 5 distinct peaks are now clearly visible also at high level. Due to a more reliable estimate of the damping ratio, a good agreement is confirmed for the third mode, and a relevant improvement is also achieved in the cases of the first two frequencies, as evidenced by the phase plot. Again by inspecting the comparison between FRF syntheses, we may conclude that mode 4 and 5 are the most distorted and, as expected, a huge discrepancy is observed in the magnitude plot.

\begin{tabular}{|c|c|c|c|c|c|}
\hline Location & Coeff. 1 & Coeff. 2 & Coeff. 3 & Coeff. 4 & Coeff. 5 \\
\hline \hline RiReBC:Z & 0.96 & 1.72 & 1.94 & 1.68 & 0.95 \\
\hline RiFrBC:Z & 0.98 & 1.68 & 1.25 & 1.78 & 1.05 \\
\hline LeReBC:Y & 0.52 & 0.85 & 0.57 & 1.06 & 0.08 \\
\hline LeFrBC:X & 0.36 & 1.00 & 0.40 & 1.64 & 0.97 \\
\hline
\end{tabular}

TABLE 4: Improved ratio between averaged real and imaginary parts in logarithmic scaling. The estimate is performed in the $15-16.5 \mathrm{~Hz}$ band. The selected poles are depicted in Fig. 12.

Again, the last step is the conversion of the reduced state-space model to the physical space. In Figure 16 the complex-valued coefficients estimated by the FNSI method in the whole frequency bandwidth are represented. With respect to Figure 14 the diagram appears highly improved. The sharpness of the peaks is now mitigated both for the real and the imaginary parts. Extremely interesting results are obtained by performing the estimate in the frequency range from $15 \mathrm{Ho} 16.5 \mathrm{~Hz}$ (see Table 4), where the ratio between the averaged real and imaginary parts, in log scale, are now positive for all the coefficients, meaning that, finally, a good estimate has been accomplished. We comment that the trend of the estimated nonlinear restoring forces 
(a)

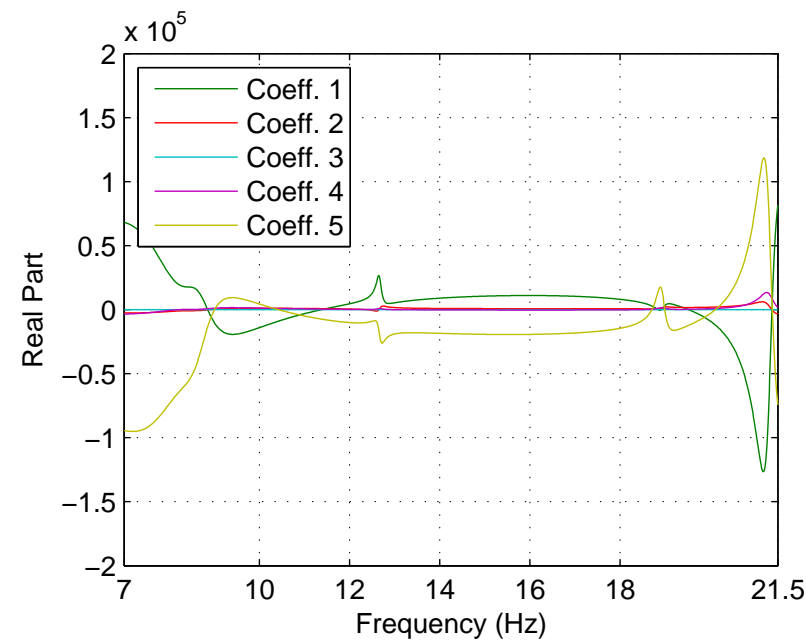

(b)

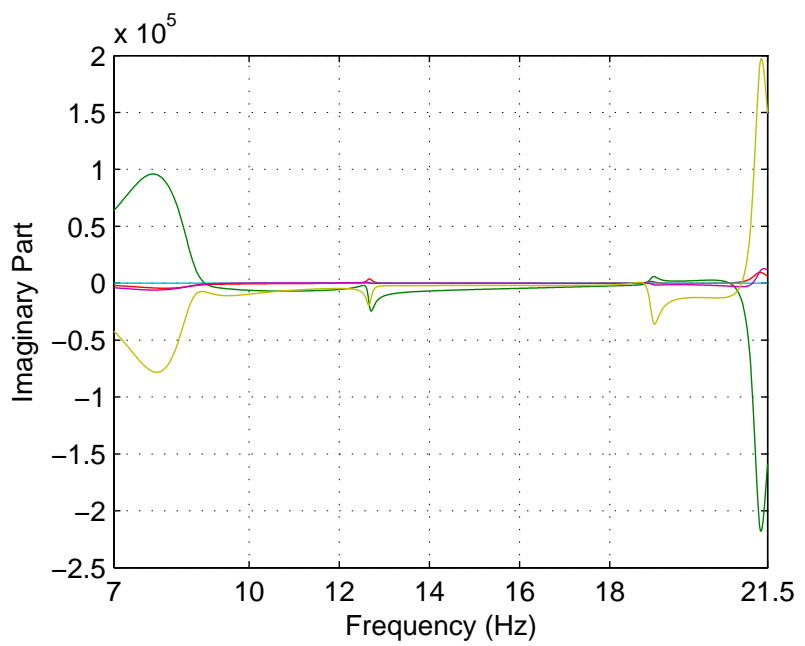

Figure 16: Improved complex-valued estimates of the 5 nonlinear coefficients related to the output LeFrBC:X in the full bandwidth $(7-21.5 \mathrm{~Hz})$. The selected poles are depicted in Fig. 12.

is consistent with the nature of the bolted connections (see Fig. 17). In fact, a combined softening-hardening behavior can be observed for RiReBC:Z, RiFrBC:Z and LeReBC:Y, while in the case of output LeFrBC:X, a simple softening effect is found out. It is worth to stress that due the high stiffness, the nonlinear behavior of bolted connections is activated in the very small scale of relative displacements $\left(10^{-2} \mathrm{~mm}\right)$. Moreover, a high density of points is present inside the softening region $\left( \pm 3 \cdot 10^{-2} \mathrm{~mm}\right)$, that may probably explain the overall softening behavior observed in the detection step (Sec. 4), and that can now certainly considered imputable to loosening in the attachments.

\section{CONCLUSIONS}

In the presented research, new tools and strategies have been implemented in the FNSI methodology to tackle the experimental identification of a full-scale aircraft, affected by the presence of many nonlinearities. The strength of the proposed comparison approach consists in the possibility of reducing the dimensionality of the inverse problem, avoiding the memory issues that may arise when large data sets are employed. On the other hand, the nonlinear identification can be carried out without loosing relevant information that might be related to the not included outputs. In addition, this strategy can be utilized to evaluate the capability of the chosen basis functions of encompassing the activated nonlinear phenomena. In this context, the limits of the stabilization analysis have been exploited at high level. A new visualization tool, named comparison diagram, is used to reveal the existence of comparable model orders in which the underlying linear properties are recovered within certain tolerance limits. At high level, cubic splines with 5 knots on the relative displacement, related to the selected outputs, are included in the FNSI routine. An improved agreement is found when comparing FRFs synthesized at low and high levels, after a novel procedure for spurious poles' removal is applied. In the end, the estimated nonlinear restoring forces have proved to be consistent with the nature of the bolted connections. Despite the fact that an overall softening behavior has been observed in the detection step, the estimates have revealed an additional combined softening-hardening behavior, that is certainly imputable to loosening in the attachments. A less satisfying agreement is found out on estimates of damping ratios at high level, that may be probably related to the activation of nonlinear phenomena involving the damping forces. In the present study splines on velocity were not considered, but this will be the focus of our future investigations. More over, to refine the achieved results effort will be spent for improving the proposed novel tools and procedures.

\section{ACKNOWLEDGMENTS}

The author J.P. Noël is a Postdoctoral Researcher of the Fonds de la Recherche Scientifique - FNRS which is gratefully acknowledged. 
(a) $\underline{\operatorname{RiReBC}: Z}$

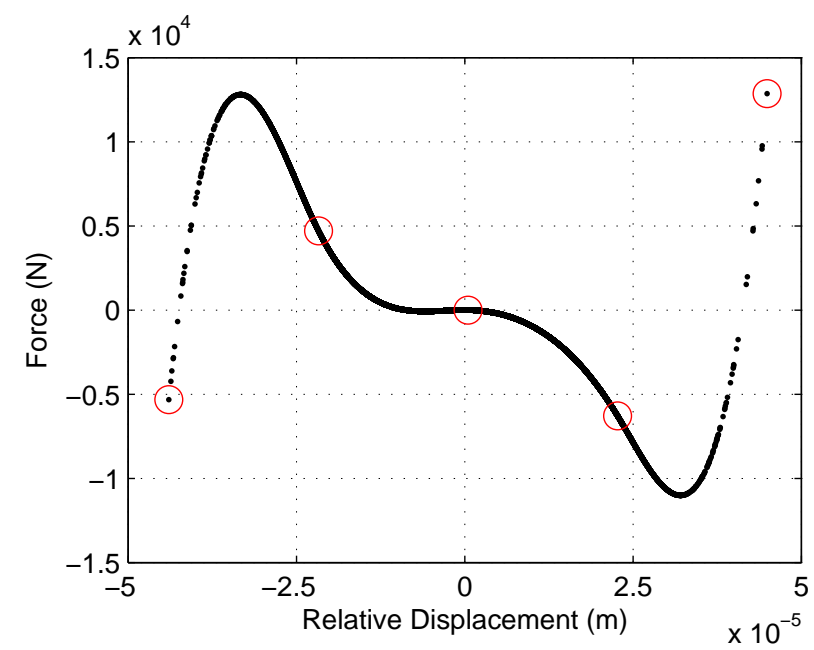

(c) LeReBC:Y

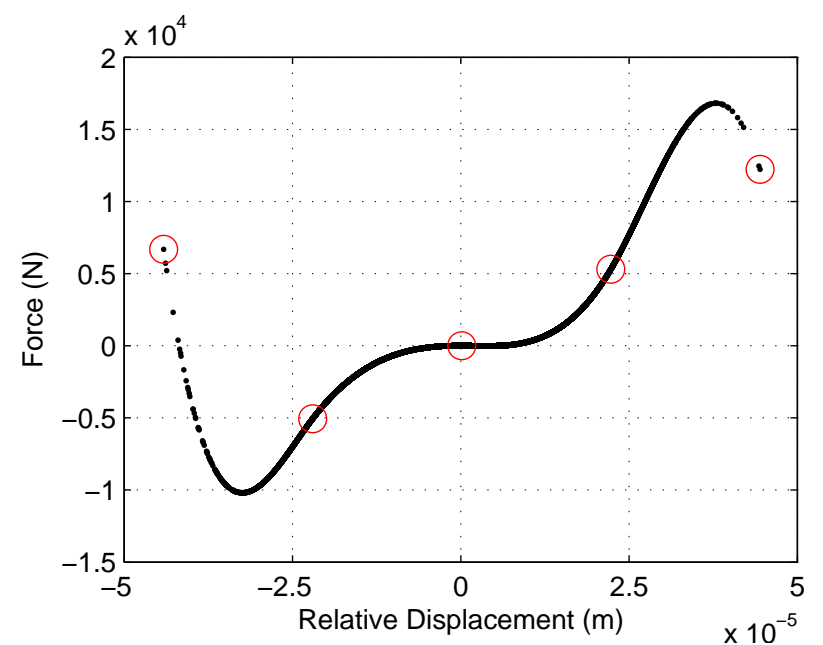

(b) $\underline{\operatorname{RiFrBC}: Z}$

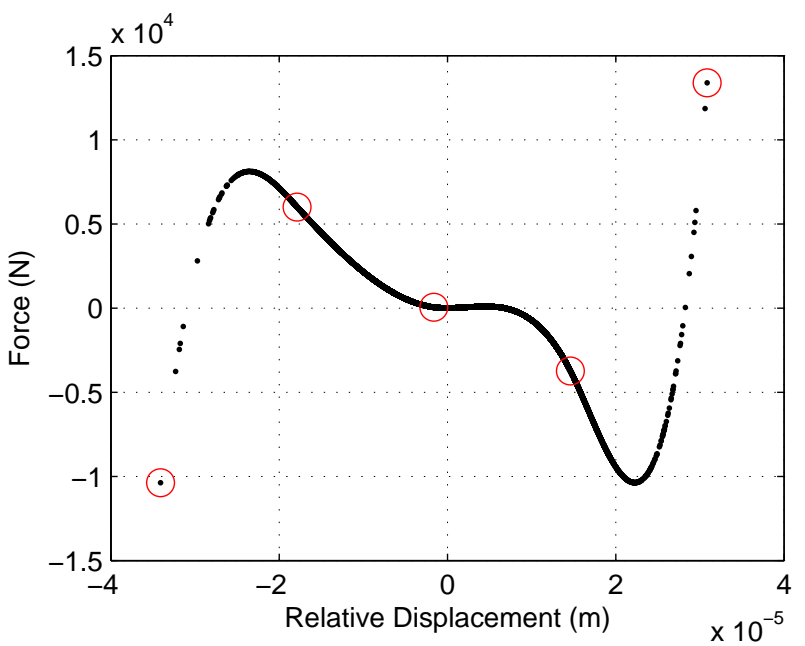

(d) LeFrBC:X

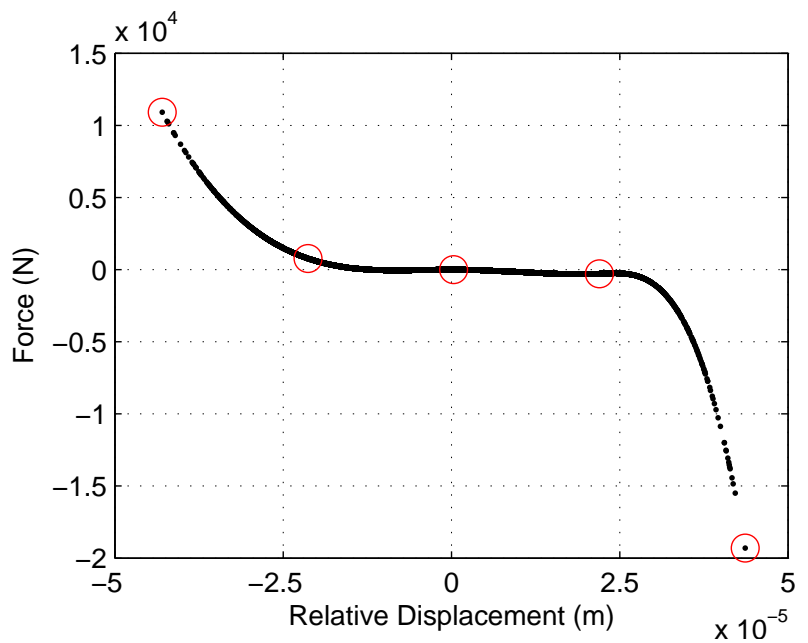

Figure 17: Improved nonlinear stiffness curves reconstructed at high level (44 N RMS) at different output locations. Red circles localise the 5 chosen knots of the splines. The selected poles are depicted in Fig. 12.

\section{REFERENCES}

[1] Ahlquist, J. R., Carreño, J. M., Climent, H., de Diego, R. and de Alba, J., Assessment of nonlinear structural response in A400M GVT, Structural Dynamics, Volume 3, pp. 1147-1155, Springer, 2011.

[2] Carrella, A. and Ewins, D., Identifying and quantifying structural nonlinearities in engineering applications from measured frequency response functions, Mechanical Systems and Signal Processing, Vol. 25, No. 3, pp. 1011 - 1027, 2011.

[3] Noël, J.-P., Renson, L., Kerschen, G., Peeters, B., Manzato, S. and Debille, J., Nonlinear dynamic analysis of an F-16 aircraft using GVT data, Proceedings of the International Forum on Aeroelasticity and Structural Dynamics, 2013.

[4] Van Overschee, P. and De Moor, B., Subspace Identification for Linear Systems: Theory, Implementation and Applications, Kluwer Academic Publishers, Dordrecht, The Netherlands, 1996.

[5] Marchesiello, S. and Garibaldi, L., A time domain approach for identifying nonlinear vibrating structures by subspace methods, Mechanical Systems and Signal Processing, Vol. 22, pp. 81-101, 2008.

[6] Noël, J. and Kerschen, G., Frequency-domain subspace identification for nonlinear mechanical systems, Mechanical Systems and Signal Processing, Vol. 40, pp. 701-717, 2013. 
[7] Noël, J., Kerschen, G., Foltête, E. and Cogan, S., Grey-box identification of a non-linear solar array structure using cubic splines, International Journal of Non-Linear Mechanics, Vol. 67, No. 0, pp. 106 - 119, 2014.

[8] Noël, J., Marchesiello, S. and Kerschen, G., Subspace-based identification of a nonlinear spacecraft in the time and frequency domains, Mechanical Systems and Signal Processing, Vol. 43, pp. 217-236, 2014.

[9] Adams, D. and Allemang, R., A frequency domain method for estimating the parameters of a non-linear structural dynamic model through feedback, Mechanical Systems and Signal Processing, Vol. 14, pp. 637-656, 2000.

[10] Peeters, B., Van der Auweraer, H., Guillaume, P. and Leuridan, J., The PolyMAX frequency-domain method: A new standard for modal parameter estimation?, Shock and Vibration, Vol. 11, No. 3-4, pp. 395-409, 2004.

[11] Heylen, W., Lammens, S. and Sas, P., Modal Analysis Theory and Testing, Katholieke Universteit Leuven, Departement Werktuigkunde, 2007.

[12] De Boor, C., A Practical Guide to Splines, Springer-Verlag, New York, 1978.

[13] Richards, C. and Singh, R., Identification of multi-degree-of-freedom non-linear systems under random excitations by the reverse path spectral method, Journal of Sound and Vibration, Vol. 213, No. 4, pp. 673-708, 1998. 